\title{
Lithium in the Coma Berenices open cluster
}

\author{
A. Ford ${ }^{1}$, R. D. Jeffries ${ }^{1}$, D. J. James ${ }^{2}$, and J. R. Barnes ${ }^{2}$ \\ 1 Department of Physics, Keele University, Keele, Staffordshire ST5 5BG, UK \\ e-mail: rdj@astro.keele.ac.uk \\ 2 School of Physics and Astronomy, University of St. Andrews, North Haugh, St Andrews, Fife, KY16 9SS, UK \\ e-mail: djj@st-andrews.ac.uk
}

Received 14 August 2000 / Accepted 12 February 2001

\begin{abstract}
Lithium abundances, radial velocities, and rotational velocities are reported for 20 candidate F, G and K stars of the sparse Coma Berenices open cluster. All the stars are proper-motion selected, and our radial velocities support the membership credentials of at least 12 of the candidates. Combining our data with that in the literature, we have a close-to-complete census of Li abundances for late-type stars with $M_{V}<5.8$. These data show that the Li-depletion pattern in Coma Ber is similar, but not identical, to that in the Hyades cluster, which has a similar age but higher metallicity. Several Coma Ber F stars have suffered significantly more Li depletion than their counterparts in the Hyades. The G and early-K stars of Coma Ber have undergone less Li depletion than those of the Hyades, but much more than that predicted by standard evolutionary models featuring only convective mixing. This provides strong evidence for additional mixing and Li depletion operating in these stars during their first $400 \mathrm{Myr}$ on the main sequence, amounting to $0.3 \mathrm{dex}$ at $6000 \mathrm{~K}$ and rising to $0.8-1.2$ dex at $5400 \mathrm{~K}$. We find that 4 of the radial-velocity non-members are among a small number of low-mass stars which were previously reported as part of an extra-tidal moving group associated with the Coma Ber cluster. As a result, we now find that the luminosity function of this moving group is indistinguishable from that of the central cluster. It is uncertain whether there may be a significant number of stars with even lower masses among the moving group.
\end{abstract}

Key words. stars: abundances - stars: late type - stars: interiors - open clusters and associations: individual: Coma Berenices

\section{Introduction}

Lithium is the only metal which was formed in significant quantities in the big bang and yet can easily be burned in stellar interiors. Knowledge of the primordial lithium abundance could, in principle, be used to constrain big-bang nucleosynthesis models and the universal baryon density (Yang et al. 1984). Li abundances in Population II stars might represent this primordial abundance (Bonifacio \& Molaro 1997), but it is debatable whether stellar mixing and processing have depleted lithium from a higher initial value (Deliyannis \& Ryan 1997; Ryan et al. 1999). Standard stellar-evolution models, incorporating only convective mixing, predict little Li depletion in Population II stars. However, non-standard processes such as microscopic diffusion, rotation-induced turbulence, or gravity waves might have reduced the surface Li by factors of 2-3 (Pinsonneault et al. 1999).

Much of the support for non-standard processes is established in observations of young clusters, where rapid rotation and loss of angular momentum may well drive

Send offprint requests to: A. Ford, e-mail: af@astro.keele.ac.uk additional mixing. The standard models cannot explain the "Boesgaard Gap" of Li-depleted mid-F stars in clusters such as the Hyades (Michaud 1986; Charbonneau \& Michaud 1988), the spread in lithium abundances in younger clusters such as the Pleiades (Soderblom et al. 1993a) and $\alpha$ Per (Balachandran et al. 1996), or the evidence that Li depletion continues in main-sequence stars, even though the bases of their convection zones (CZs) are too cool to burn Li (Jeffries \& James 1999).

Late-type stars in the Coma Berenices open cluster offer an important test of Li depletion models. With an age of 400-500 Myr (Philip et al. 1977; Lyngå 1987) Coma Ber is similar to the well studied Hyades and Praesepe clusters (ages $\simeq 600-700 \mathrm{Myr}-$ Mermilliod 1981). Importantly, these open clusters have a set of consistently-determined spectroscopic iron abundances: Coma Ber $[\mathrm{Fe} / \mathrm{H}]=$ $-0.052 \pm 0.026 ;$ Hyades $[\mathrm{Fe} / \mathrm{H}]=+0.127 \pm 0.022$; Praesepe $[\mathrm{Fe} / \mathrm{H}]=+0.038 \pm 0.039$ (Boesgaard \& Friel 1990; Friel \& Boesgaard 1992). Because the metallicity in Coma Ber is lower than that of the Hyades, its G and $\mathrm{K}$ stars should have shallower CZs at similar effective temperatures $\left(T_{\text {eff }}\right)$, and are predicted to undergo significantly less Li depletion during the pre-main-sequence (PMS) convective-mixing 
phase. As CZ-base temperatures are too cool to burn Li in main-sequence stars hotter than $\simeq 5000 \mathrm{~K}$, continuing Li depletion in these stars is not predicted by standard models. On this basis we would expect the Li-depletion patterns in these three post-ZAMS clusters to be very different, strongly dependent on metallicity, and set during their PMS evolution. Furthermore, because the Pleiades, with an age of $100 \mathrm{Myr}$, has a consistently-determined metallicity $([\mathrm{Fe} / \mathrm{H}]=-0.034 \pm 0.024)$, we can compare it with the older Coma Ber cluster to study the presence, timescales and mass dependence of any non-standard mixing and Li-depletion mechanisms for F, G and $\mathrm{K}$ stars which have reached the ZAMS.

Jeffries (1999 - hereafter J99) obtained Li abundances for a small sample of $F$ and $G$ stars in the Coma Ber cluster. It was found that the depletion pattern was very similar to that of the Hyades, with only a hint of higher Li abundances in stars cooler than $5700 \mathrm{~K}$. J99 concluded that the $\mathrm{Li}$ depletion in a range of clusters (including Blanco 1 and the Pleiades, as well as the Hyades and Coma Ber) is ordered according to the cluster age, rather than metallicity. This is contrary to the predictions of standard models. However, non-standard mixing is in addition to PMS convective mixing, so clear differences between Coma Ber and the Hyades should remain. It is, therefore, still difficult to explain the Coma Ber Li abundances unless convective PMS Li depletion is strongly inhibited in all of the sample clusters. The conclusions of J99 were tempered by the small sample size - 10 single members, 4 spectroscopic binaries and one possible nonmember - and the relatively low resolution $(0.5 \AA)$ spectra used to study blended Li lines.

Increasing the sample size is difficult because the cluster is spread over a wide area on the sky, is sparse, and has few known low-mass members. A new proper-motion study has recently been published by Odenkirchen et al. (1998, hereafter O98), who have identified several lowmass candidates which were not observed in J99. In addition, they find a core-halo spatial distribution and a co-moving group of extra-tidal stars. There is some evidence (albeit based on very small number statistics) that the mass function is still increasing towards lower masses in those stars at large distances from the cluster centre. Towards the cluster core the mass function appears sharply truncated just below $1 M_{\odot}$. These observations would agree with the idea that low-mass stars undergo mass segregation and preferential evaporation from young clusters, would explain the paucity of low-mass stars in previous surveys, and would predict a large number of stars with still lower masses at $>5^{\circ}$ from the cluster centre.

In this paper we determine radial velocities for Coma Ber cluster-member candidates proposed in $\mathrm{O} 98$ as well as a few other candidates from earlier proper-motion surveys (Trumpler 1938). We use these results to filter the candidate members, then determine Li abundances for all the objects and re-examine the conclusions of J99 using an enlarged sample of stars.

\section{Observations and analysis}

\subsection{Target selection}

The main source of new targets in this work (compared with J99) is the proper-motion survey of O98, based upon Hipparcos (Perryman et al. 1997) and ACT (Urban et al. 1998) proper-motion measurements within a $20^{\circ}$ radius of the nominal Coma Ber open cluster centre. For the brighter stars $(V<8.5)$ in the Hipparcos catalogue, proper motions alone were used to select members, whereas fainter stars $(V<10.5)$ were chosen from the ACT catalogue using proper motions in conjunction with photometric constraints. A total of 34 stars with $V<10.5$ were identified in this way (20 of them had $V<8.5$ ). This catalogue is expected to be reasonably complete, although a cross-check with the number of cluster members predicted by the excess stellar density over background counts suggests that perhaps as many as 40 per cent of members could have been missed. The reasons for this are two-fold: first, the instantaneous proper-motion measurements of binary systems may have given misleading information about their membership. This incompleteness is probably magnitude-independent. Second, for fainter targets $(V>8.5)$, the Tycho photometry used for photometric selection can be quite uncertain. This, together with the severe photometric constraints used by O98 could have led to additional incompleteness among the faintest objects.

For the main purpose of this paper incompleteness is not a great problem, except that it limits the number of objects for which we can measure Li abundance. The targets observed here were selected as proper-motion members of the cluster by $\mathrm{O} 98$ and all are F, G or K type, with $9.10<V<10.78$. Including those stars observed by J99, we have studied all the stars from O98 which are mid-F type or later. Five faint proper-motion candidates (Ta 3, Ta 13, Ta 19, Ta 20 and Ta 21) were also selected from Trumpler (1938), four of which (the exception being Ta 20) have been reported to show photometric modulation which is consistent with their identification as youthful cool cluster members by Marilli et al. (1997). These targets were faint enough $(10.36<V<11.18)$ that their absence from the catalogue of O98 does not argue strongly against their membership. A list of the new targets observed in this paper is given in Table 1.

\subsection{Spectroscopy}

The new spectroscopic data acquired for this paper were obtained at the $2.5 \mathrm{~m}$ Isaac Newton Telescope (INT) on 1998 November 26, and at the $4.2 \mathrm{~m}$ William Herschel Telescope (WHT) on 1998 November 28-29, 1998 December 06-08, and 1999 December 22-23 inclusive.

The INT data were taken with the same instrumental setup as described by J99 and analysed in exactly the same way to derive heliocentric radial velocities and the equivalent widths $(E W$ s $)$ of the Li I $6707.8 \AA /$ Fe I $6707.4 \AA$ 
blend. The instrumental resolution was $0.5 \AA$ and the data consisted of a pair of spectra for each target which were co-added to give a signal-to-noise ratio $(s / n)$ of approximately 200 per $0.22 \AA$ pixel.

The WHT data were taken with the Utrecht Echelle Spectrograph (UES) in cross-dispersing mode at the Nasmyth focus of the telescope. The detector used was a SITe1 $2048 \times 2048 \mathrm{CCD}$. The UES was set up with a 31 grooves $/ \mathrm{mm}$ grating and a central wavelength of $5875 \AA$, so that a wavelength range from $4600 \AA$ to $9000 \AA$ was covered at a dispersion of $0.066 \AA$ per $24 \mu \mathrm{m}$ pixel in the region of the $\operatorname{Li}$ I $6708 \AA$ line. The resolving power was 45000 for the 1 arcsec slit width we used.

The usual calibration frames, tungsten flat-fields and B stars were taken, as well as thorium-argon lamp exposures. The data were reduced using the Starlink ECHOMOP and FIGARO packages, which included bias-subtraction, flat-fielding, subtraction of scattered light and wavelength calibration. The $s / n$ of these spectra in the radial-velocity orders (see below) and around the Li I $6708 \AA$ line were in the range 60 to 170 per extracted spectrum pixel. Radial velocities $(R V \mathrm{~s})$ were measured using cross-correlation techniques in the spectral range 5490 to $5584 \AA$ and 5940 to $6150 \AA$, which contained many sharp, neutral metal lines and very little telluric contamination. The standard templates used to determine heliocentric $R V \mathrm{~s}$ were HD 222368, HD 32963, HD 126053 and HD 693. Spectra for several of our objects showing the region around the $6708 \AA \mathrm{Li}$ line are shown in Fig. 1 .

The $R V \mathrm{~s}$ obtained from the INT and WHT spectra are detailed in Table 1 . The errors in the INT velocities, taken with a Cassegrain spectrograph, are dominated by wavelength stability. Repeat exposures and cross-correlation of standards taken on different nights yield an estimated internal error of $\pm 2 \mathrm{~km} \mathrm{~s}^{-1}$, which is larger than the external error of putting the velocities onto the standard system. UES is mounted on a Nasmyth platform and has much higher resolution. The same experiments on standard stars observed at different times yield internal error estimates of less than $0.3 \mathrm{~km} \mathrm{~s}^{-1}$, with a likely external error of about $\pm 1 \mathrm{~km} \mathrm{~s}^{-1}$. We quote the approximate sum of internal and external errors in Table 1, as this is appropriate for comparing with other data (in particular that from J99), although this might slightly over-estimate the internal scatter within the WHT data.

Projected equatorial velocities were estimated for all the stars by cross-correlating the same spectral orders used for $R V$ determination with those of slowly-rotating, chromospherically-inactive stars of a similar spectral type. The width of the cross-correlation peak was calibrated against $v_{\mathrm{e}} \sin i$ by artificially broadening the template-star spectra with a limb-darkened rotation profile (Gray 1992) and cross-correlating against other template stars. A limbdarkening coefficient of 0.6 was used. For the INT spectra only an upper limit of $15 \mathrm{~km} \mathrm{~s}^{-1}$ could be determined. For the WHT data, with better resolution, we could measure $v_{\mathrm{e}} \sin i$ down to a limit of $6 \mathrm{~km} \mathrm{~s}^{-1}$ and several stars showed broadening greater than this. From numerical ex-

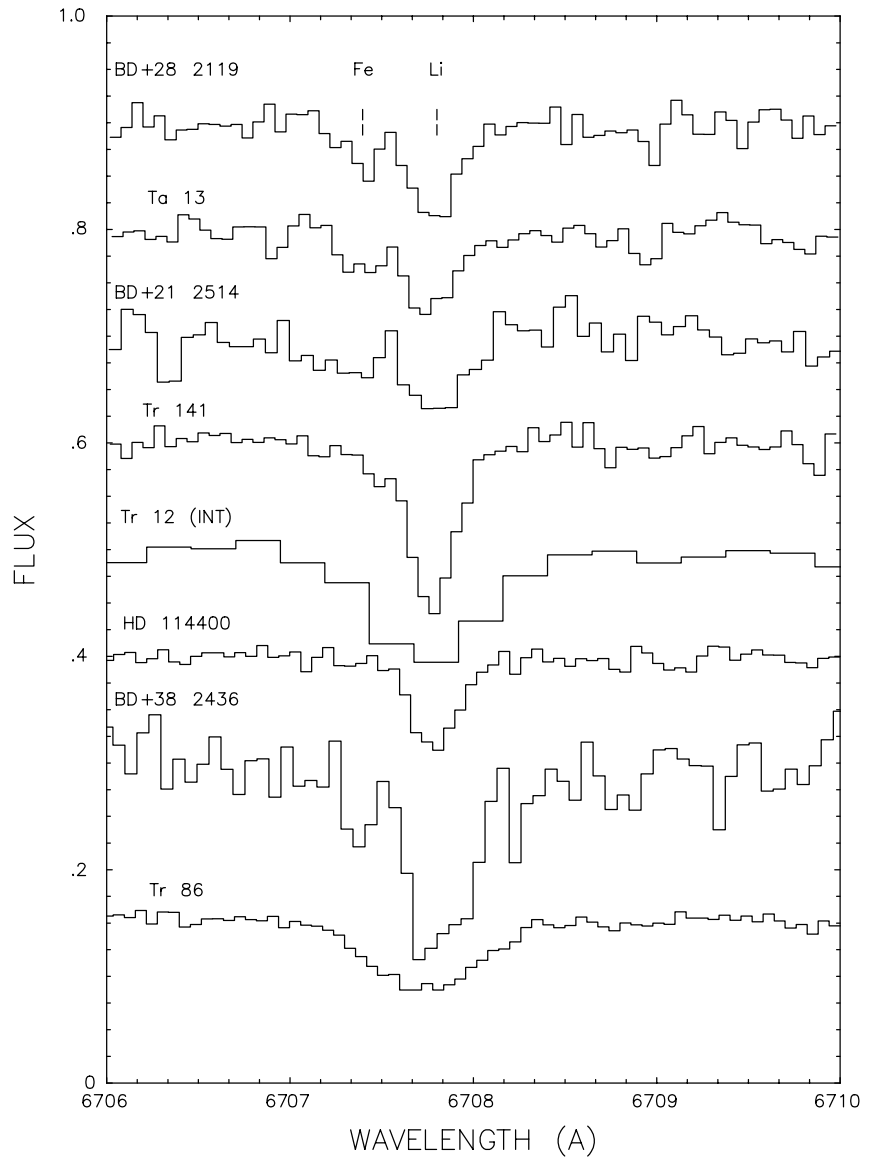

Fig. 1. Spectra in the region of the $6708 \AA$ line for a selection of our sample stars. The spectra have been normalised to unity and offset for clarity. Spectra have been corrected for their heliocentric radial velocities

periments we estimate accuracies of order $10 \%$ for these values, which are listed in Table 1.

\subsection{Lithium abundances}

The main purpose of our paper is to compare the Li abundances of cool stars in the Coma Ber open cluster with those in other clusters such as the Pleiades and Hyades. To do this is absolutely essential that consistent abundance determination techniques are used for all the stars involved in the comparison, including atmospheric models, deblending techniques and temperature scales (see Balachandran et al. 1995). Because all these data were taken with different resolutions and signal-to-noise ratios, and since only equivalent widths are available to us for most of the comparison clusters, we are forced to use curve of growth techniques for abundance estimations.

The Li I $(6706.6 \AA+6707.9 \AA)$ doublet is blended with the Fe I $6707.4 \AA$ line only in lower resolution data. Where the Li doublet and Fe lines are resolved we use equivalent widths for the Li doublet alone if quoted. For Coma Ber stars the lines are clearly resolved in most of our WHY spectra and the equivalent width is determined by fitting a synthesised $\mathrm{Li}+\mathrm{Fe}$ spectrum. Table 2 lists these $E W \mathrm{~s}$ 
along with their errors determined from chi-squared fits. For lower resolution data and for our INT Coma Ber spectra we adopt a single deblending correction to the integrated $\mathrm{Li}+\mathrm{Fe} E W$. The empirical correction we use is that the $E W$ of the Fe line (plus some even weaker CN features) is given by $20(B-V)-3 \mathrm{~m} \AA$. In our INT spectra, the total $\mathrm{Li}+\mathrm{Fe} E W$ is measured by integrating below a continuum, fitted to line-free regions around the Li doublet. These total $E W \mathrm{~s}$ (and errors from the continuum fitting and integration) are listed in Table 2 along with the deblended Li doublet $E W$ s. Note that the colour errors feed through to give a negligible additional error $(<1 \mathrm{~m} \AA)$ in the empirical deblending formula.

Of course, when dealing with clusters of differing metallicities we might expect the deblending to vary. $[\mathrm{Fe} / \mathrm{H}]$ for the Hyades is 1.5 times that of Coma Ber and we would expect the weak blended Fe line to be 1.5 times as strong as well. We choose not to try and account for this explicitly because (as we discuss further in Sect. 3.3) this effect is small compared with the differences we see between the Hyades and Coma Ber Li doublet EWs and besides, at the cool temperatures where the deblending error might contribute most, the vast majority of the spectra are taken at high resolution where the blending is not an issue.

Effective temperatures for all stars were derived from $B-V$ colours using the calibration of Saxner \& Hammarbäck (1985) for stars with $B-V<0.64$ or BöhmVitense (1981) otherwise. The Saxner \& Hammarbäck relation includes a small metallicity dependent term and we assume that a correction of the same additive form is appropriate for use with the Böhm-Vitense relation. The effective temperatures of the Coma Ber stars along with errors derived by propagating the photometry errors through the colour-temperature relation are given in Table 2. We note here that choice of effective temperature scale or the metallicity dependence of the temperature scale cannot alter our conclusions significantly. As we will see in Sect. 3.3 a change in effective temperature changes the derived $\mathrm{Li}$ abundance such that a star moves along the trend of $\mathrm{Li}$ versus effective temperature. So while absolute Li abundances for individual stars might change, the trends in abundance and inter-cluster comparisons do not. Li abundances (quoted as $\log N(\mathrm{Li})=12+\log (\mathrm{Li} / \mathrm{H})$ ) were estimated using interpolation of the LTE curves of growth provided by Soderblom et al. (1993a) - which use the appropriate gravity for the ZAMS stars considered here. Small (of order 0.05-0.1 dex) NLTE corrections were made to these using the code of Carlsson et al. (1994). The sign of these corrections varies with temperature: for hot stars it leads to a decrease in the Li abundance, while the reverse is true for cooler stars. For the Coma Ber stars the $E W \mathrm{~s}$, adopted $T_{\text {eff }}$ values and their associated errors are given in Table 2. NLTE Li abundances are also given along with an error that is derived purely by folding the $E W$ and $T_{\text {eff }}$ errors through the curves of growth. Note that these errors do not reflect the true abundance errors, which must also include contributions from uncertainties in the temperature scale, atmospheres, convection treatment, microturbulence etc. (see below). However they do represent the true levels of uncertainty for comparing relative abundances between stars in the Coma Ber and other clusters which have similar colours and Li abundances determined using the same curves of growth.

Special mention is made of $\mathrm{BD}+28$ 2119. This star has little photometry available and the Tycho $B-V$ value had very large errors $(0.876 \pm 0.125)$. We measured the strength of several Fe I lines in the $6708 \AA$ region for all the member stars with WHT spectra and plotted them against $B-V$. The resulting graphs seem to indicate that the true $B-V$ for $\mathrm{BD}+282119$ (assuming it to be a typical cluster star) is closer to $0.79 \pm 0.02$ and this is what we use to calculate the $\mathrm{Li}$ abundance.

In Sects. 4.1 and 4.2 we compare the Li abundances in Coma Ber (and other clusters) with theoretical models of $\mathrm{Li}$ depletion. For this purpose it is important to have an idea of the accuracy of the absolute Li abundances, in addition to the precision of the relative $\mathrm{Li}$ abundances. We expect larger, systematic errors in the absolute Li abundances than the uncertainties listed in Table 2. This is because we must include contributions from uncertain temperature scales, microturbulence and the fact that different atmospheres and convection models will yield slightly different abundances. Changes in the atmospheric assumptions do change the abundances in all the clusters systematically with respect to the theoretical models. We have experimented with this by performing a spectral synthesis for our best WHT spectra using Kurucz ATLAs9 model atmospheres (Kurucz 1993) using different convection models (mixing length theory with and without overshoot or full spectrum turbulence), different microturbulence parameters and temperatures differing by $\pm 100 \mathrm{~K}$.

We find that the abundances we determine are within 0.1 dex of the abundances determined from curve of growth techniques and that alteration of the atmospheric assumptions also causes $\mathrm{Li}$ abundances changes of $0.1 \mathrm{dex}$ or less. We conclude that for the purposes of comparison with theoretical models, systematic errors of about 0.1 dex in the Li abundance should be added to the relative errors listed in Table 2.

\section{Results}

\subsection{Radial velocities}

A summary of the heliocentric $R V$ measurements found in this paper, along with others from the literature, is given in Table 1 . We take the range of $R V \mathrm{~s}$ for likely cluster members to be $2 \sigma$ from $\pm 1 \mathrm{kms}^{-1}$ (O98), which is a typical cluster velocity dispersion (Rosvick et al. 1992). The cluster convergent point is sufficiently distant that any change in $R V$ with position will be negligible. We suggest that those objects with radial velocities just outside our range are likely to be widely-spaced spectroscopic binaries which are members of the cluster. This is the case for 
Tr 102, which has also been identified as an SB1 star by Bounatiro (1993).

The combination of proper-motion, photometric, and radial-velocity selection make it very unlikely that we have wrongly classified non-members as cluster stars. For example, O98 estimate a contamination level of less than 5 per cent among their brightest stars (with Hipparcos proper motions), based on proper-motion measurements alone. For fainter stars with less-accurate ACT proper motions this probability is likely to be much larger (evidence presented in Sect. 4.3 suggests it could be as high as 40 per cent). However, if we assume field stars have $R V \mathrm{~s}$ which are randomly distributed between $\pm 30 \mathrm{~km} \mathrm{~s}^{-1}$ (Wielen 1977), then there is only a $\sim 10$ per cent chance of mistaking a non-member for a cluster member using the $R V$ classification alone, and this will shrink drastically when we combine it with proper-motion and photometric selection.

We stress that our assessment of membership is based only on photometric, proper-motion and radial-velocity considerations. In particular we do not consider the lithium abundance as a defining characteristic. However, most of the stars which showed very discrepant $R V \mathrm{~s}$ (in this paper and J99) showed either very little or no lithium compared with candidate members of similar $T_{\text {eff }}$ (see Sect. 3.3). The exceptions here are the candidate SB1 system, $\operatorname{Tr} 102$, and the $R V$ non-member, Ta 21, which do show Li abundances consistent with those of other cluster members. Ta 21 may be a close binary system in the Coma Ber cluster and warrants further $R V$ investigation, but we conservatively treat it as a non-member in the rest of this paper. We suspect that none of the remaining $R V$ non-members are likely to have discrepant velocities as a result of being members of close, tidally-locked binaries, because such systems usually have the same, or even enhanced, Li relative to single cluster stars (e.g. those in the Hyades - Thorburn et al. 1993). We note that Argue \& Kenworthy (1969) rejected Ta 3 and Ta 19 (selected as candidate proper-motion members by Trumpler 1938) as cluster members on the basis of their proper-motion measurements. We find that both these stars also have $R V \mathrm{~s}$ which are inconsistent with cluster membership, so the photometric modulation observed by Marilli et al. (1997) must identify them as moderately-youthful disk field stars.

\subsection{Projected equatorial velocities}

For the 20 stars in our sample, all have $v_{\mathrm{e}} \sin i \leq$ $20 \mathrm{kms}^{-1}$, and at least 11 have $v_{\mathrm{e}} \sin i \leq 6 \mathrm{kms}^{-1}$. Combining the Coma Ber cluster candidates from Table 1 with those from J99 (where all candidates had $v_{e} \sin i \leq$ $15 \mathrm{~km} \mathrm{~s}^{-1}$ ) and the $\mathrm{F}$ stars with $v_{\mathrm{e}} \sin i$ measurements in Kraft (1967), we can compare rotation rates in Coma Ber with those in the Hyades. The Hyades measurements are either $v_{\mathrm{e}} \sin i$ values from Kraft (1965), or actual equatorial velocities derived from rotation periods by Radick et al. (1987). The comparison is shown in Fig. 2 as a func-

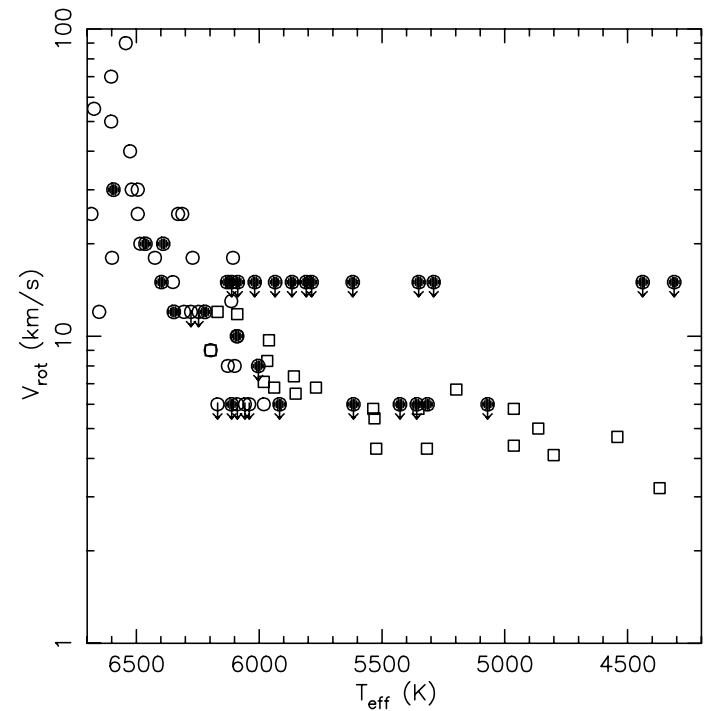

Fig. 2. Rotational velocity versus $T_{\text {eff }}$ for Coma Ber (filled circles) and Hyades (open circles - lower limits to true equatorial rotational velocity, Kraft 1965; squares - rotational velocity calculated from rotation period and assumed radius, Radick et al. 1987)

tion of $T_{\text {eff }}$ (calculated from $B-V$ and $[\mathrm{Fe} / \mathrm{H}]$ as described in Sect. 2.3), and reveals no evidence for a difference in rotation rates between Coma Ber and the Hyades. The equatorial velocities in the Hyades show little scatter at a given $T_{\text {eff }}$ and appear to decrease smoothly from about $20-30 \mathrm{~km} \mathrm{~s}^{-1}$ in mid-F stars $\left(T_{\text {eff }}=6500 \mathrm{~K}\right)$ to $10 \mathrm{~km} \mathrm{~s}^{-1}$ in late-F stars $\left(T_{\text {eff }}=6100 \mathrm{~K}\right)$, and $\sim 5 \mathrm{~km} \mathrm{~s}^{-1}$ in late$\mathrm{G} /$ early-K stars $\left(T_{\text {eff }}=5000-5500 \mathrm{~K}\right)$. Recalling that the projected equatorial velocities we observe have an extra (presumably random) $\sin i$ term factored in, then the predominance of upper limits in our data is quite consistent with the Hyades data.

\subsection{Lithium}

The photometry, Li + Fe $E W \mathrm{~s}$, deblended $E W \mathrm{~s}$, assumed $T_{\text {eff }}$ values, and calculated $\mathrm{Li}$ abundance for each star are given in Table 2. Errors in the Li abundances take into account random errors in both the photometry and $\mathrm{Li}$ $E W$ s. These are internal errors and additional external errors of \pm 0.1 dex are appropriate when comparing with $\mathrm{Li}$ abundances determined using different techniques. Three of our measurements can be compared with those of J99 (based on spectra obtained with the INT in an identical configuration to that used here):

- HIP 61205 (Bou 50). Our measurement for this star was also taken on the INT, and is in excellent agreement with the value from J99. This suggests that there is no systematic difference between our reduction and that of J99, at least for the INT spectra;

- Ta 13. The deblended Li $E W$ estimated in J99 is $28 \pm 3 \mathrm{~mA}$, in excellent agreement with the measured 
Table 1. Heliocentric radial velocity and projected equatorial velocity measurements from this and previous papers

\begin{tabular}{|c|c|c|c|c|c|c|}
\hline Object & $\begin{array}{l}\text { Other } \\
\text { Names }\end{array}$ & $\begin{array}{l}R V \text { from this } \\
\text { paper }\left(\mathrm{km} \mathrm{s}^{-1}\right)\end{array}$ & $\begin{array}{l}\text { Other } R V \mathrm{~s} \\
\text { and references }\end{array}$ & $\begin{array}{l}v_{\mathrm{e}} \sin i \\
\left(\mathrm{~km} \mathrm{~s}^{-1}\right)\end{array}$ & Status & Source \\
\hline $\mathrm{BD}+162505$ & & $-11.8 \pm 2.0$ & & $<15$ & NM & INT98 \\
\hline $\mathrm{BD}+212514$ & & $-0.6 \pm 2.0$ & & $<15$ & M & INT98 \\
\hline $\operatorname{Tr} 12$ & $\mathrm{BD}+26$ 2314, HD 105863 & $+1.2 \pm 2.0$ & $+0.43(\mathrm{a})$ & $<15$ & M & INT98 \\
\hline HD 114400 & $\mathrm{BD}+342398$ & $-1.7 \pm 2.0$ & & $<15$ & M & INT98 \\
\hline HIP 61205 & BD+36 2278, Bou 50 & $-0.5 \pm 2.0$ & $+1.1(\mathrm{~d})$ & $<15$ & M & INT98 \\
\hline $\mathrm{BD}+212514$ & & $0.0 \pm 1.0$ & & $<6$ & M & WHT98 \\
\hline $\mathrm{BD}+252631$ & & $+13.7 \pm 1.0$ & & $<6$ & $\mathrm{NM}$ & WHT98 \\
\hline Ta 13 & $\mathrm{BD}+262342, \operatorname{Tr} 213$ & $+0.9 \pm 1.0$ & $-1.6(\mathrm{~d})$ & $<6$ & M & WHT98 \\
\hline $\operatorname{Tr} 102$ & $\mathrm{BD}+272121$ & $-3.1 \pm 1.0$ & $-14, \operatorname{var}(\mathrm{a}),-14(\mathrm{c})$ & $<6$ & SB1 & WHT98 \\
\hline Ta 20 & $\mathrm{BD}+272139, \operatorname{Tr} 220$ & $+0.5 \pm 1.0$ & $+0.88(\mathrm{a}),+4.0(\mathrm{~d})$ & $<6$ & $\mathrm{M}$ & WHT98 \\
\hline $\mathrm{BD}+282119$ & & $+2.0 \pm 1.0$ & & 6 & M & WHT98 \\
\hline $\operatorname{Tr} 192$ & $\mathrm{BD}+28$ 2134, HD 109483 & $-1.3 \pm 1.0$ & $-2.4(\mathrm{~b})$ & $<8$ & M & WHT98 \\
\hline $\operatorname{Tr} 141$ & $\mathrm{BD}+292290$ & $+0.6 \pm 1.0$ & & $<6$ & $\mathrm{M}$ & WHT98 \\
\hline $\mathrm{BD}+362312$ & & $-55.6 \pm 1.0$ & & 8 & NM & WHT98 \\
\hline $\mathrm{BD}+382436$ & & $-2.0 \pm 1.0$ & & $<6$ & $\mathrm{M}$ & WHT98 \\
\hline Tyc 253417151 & & $-36.4 \pm 1.0$ & & $<6$ & $\mathrm{NM}$ & WHT98 \\
\hline HD 111878 & $\mathrm{BD}+262402$ & $-2.4 \pm 1.0$ & $-7.5(\mathrm{~b})$ & 10 & $\mathrm{M}$ & WHT99 \\
\hline $\operatorname{Tr} 86$ & $\mathrm{BD}+28$ 2109, HD 107611 & $-1.0 \pm 1.0$ & $+2.4(\mathrm{a}),-0.7(\mathrm{c})$ & 20 & M & WHT99 \\
\hline Та 3 & $\operatorname{Tr} 203$ & $-15.9 \pm 1.0$ & & $<6$ & NM & WHT99 \\
\hline Ta 19 & $\operatorname{Tr} 219$ & $-14.9 \pm 1.0$ & & $<6$ & $\mathrm{NM}$ & WHT99 \\
\hline Ta 21 & $\operatorname{Tr} 221$ & $+30.6 \pm 1.0$ & & $<6$ & SB1? & WHT99 \\
\hline
\end{tabular}

References: (a) Bounatiro (1993); (b) Knude (1989); (c) Duflot et al. (1995); (d) Jeffries (1999). Status: M - Member, NM - Non-member, SB1 - Spectroscopic Binary.

Table 2. Photometry, blended ( $\mathrm{Li}$ I + Fe I) and deblended Li I $6708 \AA E W$, assumed $T_{\text {eff }}$ values and Li abundances of stars in Coma Berenices. For the purposes of comparison with theoretical models, systematic errors of about 0.1 dex in the Li abundance should be added to the relative errors listed in Table 2

\begin{tabular}{|c|c|c|c|c|c|c|c|}
\hline Object & $V$ & $B-V$ & $\begin{array}{l}\text { Phot. } \\
\text { Ref. }\end{array}$ & $\begin{array}{l}\text { Blended } \\
E W(\mathrm{~m} \AA)\end{array}$ & $\begin{array}{l}\text { Deblended } \\
\text { Li I } E W(\mathrm{~m} \AA)\end{array}$ & $\begin{array}{l}T_{\text {eff }} \\
(\mathrm{K}) \\
\end{array}$ & $\log N(\mathrm{Li})$ \\
\hline $\mathrm{BD}+162505$ & 9.62 & $0.658 \pm 0.036$ & tyc & $11 \pm 3$ & $\leq 7$ & 5683 & $\leq 1.32$ \\
\hline $\mathrm{BD}+212514^{(*)}$ & 10.12 & $0.747 \pm 0.041$ & tyc & $\ldots$ & $\overline{31.1} \pm 1.7$ & 5427 & $1.78_{-0.10}^{+0.10}$ \\
\hline $\mathrm{BD}+212514^{(\dagger)}$ & 10.12 & $0.747 \pm 0.041$ & tyc & $56 \pm 5$ & 44.1 & 5427 & $1.93_{-0.11}^{+0.11}$ \\
\hline BD+25 2631 & 10.48 & $0.848 \pm 0.086$ & tyc & $\ldots$ & $\leq 3.5$ & 5170 & $\leq 0.55$ \\
\hline HD 111878 & 8.87 & $0.546 \pm 0.015$ & hip & $85.0 \pm 1.5$ & $\overline{77.1}$ & 6091 & $2.73_{-0.04}^{+0.04}$ \\
\hline $\operatorname{Tr} 12$ & 9.54 & $0.624 \pm 0.002$ & hip & $82 \pm 4$ & 72.5 & 5808 & $2.49_{-0.03}^{+0.03}$ \\
\hline Ta 13 & 10.51 & $0.773 \pm 0.017$ & $\mathrm{~d}$ & & $25.0 \pm 1.0$ & 5358 & $1.62_{-0.04}^{+0.04}$ \\
\hline $\operatorname{Tr} 102$ & 9.36 & $0.594 \pm 0.005$ & d & $59.6 \pm 1.4$ & 49.7 & 5917 & $2.38_{-0.02}^{+0.02}$ \\
\hline Ta 20 & 10.78 & $0.89 \pm 0.01$ & $\mathrm{a}$ & $\ldots$ & $\leq 6.0$ & 5070 & $\leq 0.69$ \\
\hline $\operatorname{Tr} 86$ & 8.50 & $0.463 \pm 0.005$ & d & $44.0 \pm 1.5$ & 37.8 & 6391 & $2.60_{-0.02}^{+0.02}$ \\
\hline $\mathrm{BD}+282119$ & 10.50 & $0.79 \pm 0.02$ & $\mathrm{e}$ & $\ldots$ & $28.8 \pm 1.0$ & 5316 & $1.64_{-0.05}^{+0.05}$ \\
\hline $\operatorname{Tr} 192$ & 9.07 & $0.57 \pm 0.03$ & tyc & $\ldots$ & $69.8 \pm 1.7$ & 6003 & $2.62_{-0.06}^{+0.06}$ \\
\hline $\operatorname{Tr} 141$ & 9.72 & $0.71 \pm 0.06$ & tyc & $\ldots$ & $45.0 \pm 1.2$ & 5616 & $2.04_{-0.12}^{+0.12}$ \\
\hline HD 114400 & 9.59 & $0.608 \pm 0.03$ & tyc & $66 \pm 4$ & 57.2 & 5866 & $2.41_{-0.09}^{+0.09}$ \\
\hline HIP 61205 & 9.71 & $0.63 \pm 0.01$ & $\mathrm{a}$ & $77 \pm 4$ & 67.4 & 5786 & $2.43_{-0.04}^{+0.05}$ \\
\hline $\mathrm{BD}+362312$ & 10.47 & $0.86 \pm 0.01$ & $\mathrm{~b}$ & $\ldots$ & $\leq 3$ & 5142 & $\leq 0.45$ \\
\hline $\mathrm{BD}+382436$ & 9.10 & $0.54 \pm 0.01$ & $\mathrm{c}$ & $\ldots$ & $\overline{80} .7 \pm 2.0$ & 6112 & $\overline{2.77}{ }_{-0.03}^{+0.03}$ \\
\hline Тa 3 & 10.77 & $0.85 \pm 0.01$ & $\mathrm{a}$ & $\ldots$ & $\leq 5.8$ & 5167 & $\leq 0.77$ \\
\hline Ta 19 & 11.18 & $0.82 \pm 0.01$ & $\mathrm{a}$ & $\ldots$ & $\leq 5.6$ & 5240 & $\leq 0.83$ \\
\hline Тa 21 & 10.36 & $0.81 \pm 0.01$ & $\mathrm{a}$ & $\ldots$ & $\overline{15} \pm 2.0$ & 5265 & $1.30_{-0.02}^{+0.02}$ \\
\hline Тус 253417151 & 10.42 & $0.797 \pm 0.078$ & tyc & $\ldots$ & $\leq 4.4$ & 5298 & $\leq 0.77$ \\
\hline
\end{tabular}

References: (a) Mermilliod (1976); (b) Haagkvist \& Oja (1973) ; (c) Oja (1985); (d) Johnson \& Knuckles (1955); (e) see Sect. 2.3; hip - Hipparcos catalogue; tyc - Tycho catalogue; ${ }^{*}$ WHT spectrum; ${ }^{\dagger}$ INT $^{\text {spectrum. }}$ 
WHT Li $E W$ in this paper. This gives some support for the accuracy of the empirical deblending performed both in this paper and in J99. However, we also note that in the case of $\mathrm{BD}+212514$, for which we have obtained both INT and WHT spectra, there is a discrepancy of $(14.9 \pm 5.3) \mathrm{m} \AA$ between the empiricallydeblended INT Li $E W$ and the measured WHT Li $E W$. The question of the detailed accuracy of the empirical-deblending process is still open and we have much more faith in the measured $\mathrm{Li} E W \mathrm{~s}$ from the higher resolution WHT spectra. But, as discussed in Sect. 3.2 of J99, errors in the empirical deblending will not alter the conclusions in that paper or this one;

- Ta 20. The higher resolution WHT spectra have allowed us to obtain a new upper limit value for the Li I $6708 \AA E W$ for this star, which is smaller than the previous value by $6 \mathrm{~m} \AA$.

A plot of lithium abundance versus $T_{\text {eff }}$ for the Coma Berenices cluster members is shown in Fig. 3, where we have added the points from J99 (favouring measurements done at higher resolution on the WHT), and supplemented these with additional $\mathrm{F}$ stars observed by Boesgaard (1987). Open symbols represent those candidate members we assume to be in binary systems. Comparison is made with similar data for stars in the Hyades and Pleiades clusters. Li $E W \mathrm{~s}$ and photometry were obtained from Boesgaard \& Budge (1988), Soderblom et al. (1993b), Thorburn et al. (1993), and Soderblom et al. (1995). In these comparison samples we have included only those stars with no evidence for close binarity. In all cases, where necessary, we first deblended the Li line and then obtained $\mathrm{Li}$ abundances using the same temperature scale and curves of growth as for the Coma Ber data.

Figure 3 shows that Coma Ber stars with spectral types earlier than that of the Sun $\left(5800 \mathrm{~K}<T_{\text {eff }}<6700 \mathrm{~K}\right)$ are clearly Li-depleted when compared with the Pleiades. This depletion is most dramatic at around $6600 \mathrm{~K}$, the "Boesgaard gap", which is more clearly seen in the Hyades data. The abundances in the Coma Ber F and early-G stars appear similar to those in the Hyades, except for a suspicion that some Coma Ber stars with temperatures around 6100-6400 K may exhibit 0.3-0.8 dex more depletion than their Hyades counterparts, although this comparison is hampered by small numbers. However, there are three Coma Ber stars which have clearly depleted their lithium abundances below the Hyades level. The Hyades stars show little evidence for a lithium abundance dispersion at a given temperature in this region, but the presence of some single and probable-binary stars with $\mathrm{Li}$ abundances similar to those of Hyades stars indicates a large spread in these abundance values among the late-F stars of Coma Ber.

The three late-F low-Li Coma Ber stars are Tr 58 , $\operatorname{Tr} 76$ and $\operatorname{Tr} 92$. Tr 58 has consistently measured abundances given in both J99 and Boesgaard (1987). Tr 76 has consistently low Li measured by J99, Boesgaard (1987) and Soderblom et al. (1990). Tr 92 has consistent mea-

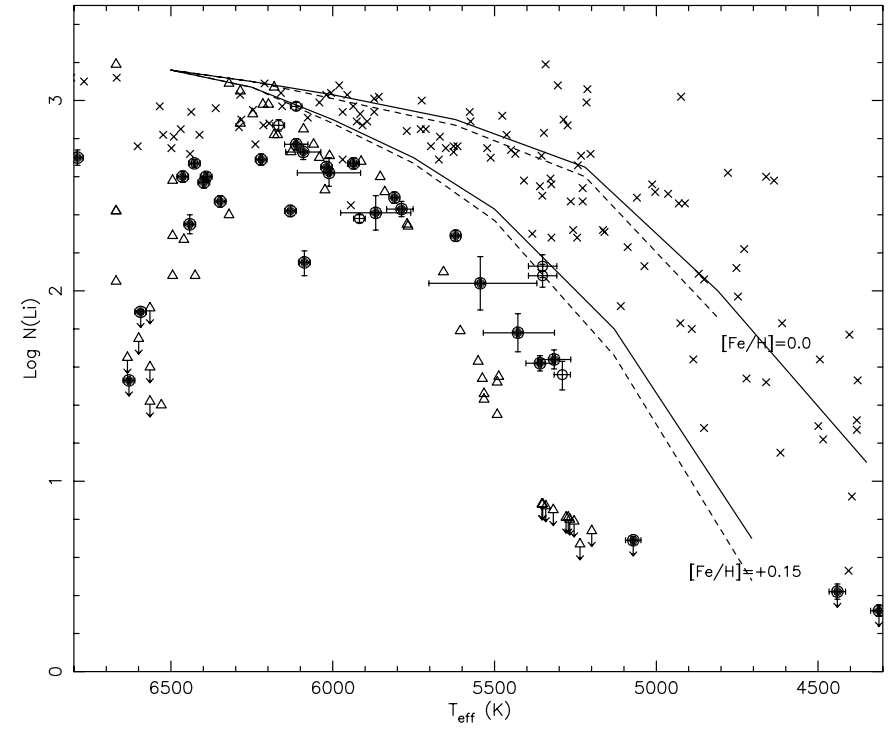

Fig. 3. NLTE Li abundances versus $T_{\text {eff }}$ for Coma Ber (filled circles - single stars, open circles - spectroscopic binaries), Hyades (triangles) and Pleiades (crosses) open clusters. The two solid lines are standard model isochrones of Li depletion (from Pinsonneault 1997) for and age of $100 \mathrm{Myr}$ and $[\mathrm{Fe} / \mathrm{H}]$ of 0.0 (upper) and +0.15 (lower). The dashed lines are as above but for an age of $700 \mathrm{Myr}$

surements in both Boesgaard (1987) and Soderblom et al. (1990). Either there is a genuine large spread in Li depletion for late-F stars in Coma Ber (which is not seen in the Hyades) or these three stars coincidentally show proper motions, photometry and radial velocities consistent with cluster membership - which we think very unlikely.

For stars with $T_{\text {eff }}<5800 \mathrm{~K}$, the addition of several new Coma Ber members adds weight to the tentative finding by J99, that the abundances of Coma Ber stars are close to, but have suffered less Li depletion than, stars of similar $T_{\text {eff }}$ in the Hyades. This discrepancy widens at lower $T_{\text {eff }}$. The reader might imagine that uncertainties in the metallicities of the two clusters, combined with the metallicity dependence of the $T_{\text {eff- }}(B-V)$ relation, could partially account for the difference. At $B-V=0.8$ the Hyades stars are around $100 \mathrm{~K}$ hotter than those in Coma Ber for the metallicities we have assumed. However, if the Coma Ber temperatures were higher, the derived Li abundances would also increase. The net effect would be to move the points almost parallel to the trend observed in the $\mathrm{G}$ and $\mathrm{K}$ stars of Coma Ber and the Hyades, thus making little difference to the comparison. A further objection could be that the empirical deblending formula used for the INT data must surely be metallicity dependent. Of course Coma Ber has the lower metallicity, so the contribution to the blended $\mathrm{Li}+\mathrm{Fe}$ line should be smaller than for the Hyades, by a factor of around 1.5 for this weak line. This would have a small effect in the sense of widening the discrepancy between Coma Ber and the Hyades. Besides, we now have higher resolution data for most of the cool stars, where the line blend is resolved (see Fig. 1). Therefore, we believe that this difference in Li depletion 
is real and well established, since it is now supported by several more objects.

\section{Discussion}

For the purposes of discussion we divide our sample into two temperature ranges. We stress that the Li abundances listed in Table 2 (and for stars in other clusters) have been determined using consistent methods. Thus intercomparison of the cluster samples need only consider internal errors due to photometry and $E W$ uncertainties. However, where we make comparison with theoretical models it must be accepted that there is an additional $\sim \pm 0.1$ dex error in the absolute Li abundances. In addition we assume that all clusters were born with the same initial $\mathrm{Li}$ abundance, but variations of \pm 0.1 dex are possible.

\subsection{F-stars $\left(5800 \leq T_{\text {eff }} \leq 6800 \mathrm{~K}\right)$}

What expectations do we have for the Li depletion patterns of $\mathrm{F}$ stars in clusters of similar age but differing metallicity? Our observations suggest that there is little difference in the position of the centre of the Boesgaard gap or its cool wing. This is in agreement with the work done by Balachandran (1995), who showed that there was essentially no difference between the Hyades and Praesepe F-star Li-depletion patterns, despite a small metallicity difference between the clusters. We do, however, see some evidence for additional $\mathrm{Li}$ depletion, relative to the Hyades (and Praesepe), among some (but not all) Coma Ber stars with temperatures in the range $6100-6300 \mathrm{~K}$.

Standard models predict that we should see almost no difference in the Li- $T_{\text {eff }}$ trends of the Hyades, Praesepe or Coma Ber F stars, since their CZs are thin enough that convective PMS Li destruction amounts to only $\sim 0.1$ dex. Still smaller discrepancies are seen between models with metallicity differences of 0.1-0.2 dex (Pinsonneault 1997). However, we see significant Li depletion among the F stars (6000-6700K) in both the Hyades and Coma Ber ranging from 0.3 dex to $>1.5$ dex in the Boesgaard gap (for an assumed initial $\log N(\mathrm{Li})$ of 3.2$)$. This depletion, which is extra to the standard model predictions, demands nonstandard effects such as microscopic diffusion or slow (nonconvective) mixing caused by rotation or gravity waves.

Boesgaard (1991) presented an interesting argument, suggesting that for stars of a given age which are in the temperature range 5950-6350 K, greater Li depletion would be seen in lower metallicity objects because the thinner CZs should make microscopic diffusion more efficient. This would lead us to expect significantly greater depletion in the Coma Ber stars compared to those of the Hyades. This is apparently what we are seeing and a $\log N(\mathrm{Li})$ value of 2.7 in the late-F stars of Coma Ber would be in reasonable agreement with the empirical relationship between $\mathrm{Li}$ abundance, age, and metallicity put forward by Boesgaard. Whilst we confirm this empirical trend, we do not believe that Boesgaard's explanation is correct. No account is taken of the fact that for late-type stars of a given temperature, a lower metallicity star has a lower mass and a relatively thicker CZ. These two effects almost cancel out for late-F stars, so that stars of a given $T_{\text {eff }}$ have similar CZ thicknesses. It is thus very unclear how a small change in metallicity affects Li depletion in the context of the microscopic diffusion model and it would be interesting to see some numerical predictions.

The work of Deliyannis et al. (1998), argues strongly against the effectiveness of diffusion in late-F stars. Instead, their observations of simultaneous $\mathrm{Li}$ and $\mathrm{Be}$ depletion in field $\mathrm{F}$ stars favour rotationally-driven mixing occuring between the $\mathrm{CZ}$ base and regions where the temperature is high enough to burn Li. Models incorporating rotational mixing are described by Pinsonneault et al. (1999). These show that in the range 6000-6500 K, the predicted depletion by the age of the Hyades is modestly metallicity dependent, with about 0.1-0.2 dex less depletion in Praesepe than in the Hyades. Intuitively this arises because at a given $T_{\text {eff }}$, the $\mathrm{CZ}$ base is cooler in lower metallicity stars, so material has to be mixed further to deplete Li. Extrapolating, we might expect even less depletion in Coma Ber stars at the same temperature, so the observed Li abundances do not seem to agree with the predictions for rotational mixing. However, more important than metallicity in these models is the rotational history of the stars themselves. Stars which begin life rotating more rapidly are able to deplete their Li faster. Hence a wide spread in initial rotation rates can lead to a wide spread in $\mathrm{Li}$ depletion at later times. We could hypothesize that the Coma Ber stars began life rotating slightly faster on average than Hyades stars, and with a larger spread in initial angular momentum.

The current rotation rate data in these clusters (shown in Fig. 2) neither support nor contradict this hypothesis. The suggestion is difficult to test because: (a) we only have $v_{\mathrm{e}} \sin i$ measurements in Coma Ber, many of which are upper limits. However, the three anomalously low Li F stars in Coma Ber do not have anomalously high $v_{\mathrm{e}} \sin i$; (b) magnetic braking causes rotation rates to converge among stars with outer convection zones, so a lack of a rotation-rate spread now, does not mean that one never existed. Certainly, spreads in Li abundance are clear among the $\mathrm{F}$ and $\mathrm{G}$ stars of older clusters such as M 67 (Jones et al. 1999), and one possible explanation which has been put forward is differing Li depletion rates caused by different rotational histories.

\section{2. $G$ and $K$ stars $\left(T_{\text {eff }} \leq 5800 \mathrm{~K}\right)$}

Our observations of $\mathrm{G}$ and $\mathrm{K}$ stars in the Coma Ber cluster cannot be adequately explained by either standard or nonstandard models. Standard models predict that almost all depletion in these stars should happen on the PMS, with no further Li destruction once stars reach the ZAMS (Pinsonneault 1997 and Fig. 3). The amount of PMS depletion will be highly dependent on the metallicity of the star: metal-rich stars will have deeper CZs and therefore 
deplete Li more efficiently than lower-metallicity stars of the same temperature. Recalling the metallicities of Coma Ber $(-0.052 \pm 0.026)$, the Pleiades $(-0.034 \pm 0.024)$ and the Hyades $(+0.127 \pm 0.022)$, this would mean that the Coma Ber lithium abundances should be close to those of similar stars in the Pleiades, due to their comparable metallicities. To illustrate the dependence of PMS depletion on metallicity, Fig. 3 shows two sets of Li depletion isochrones with a metallicity difference of 0.15 dex, approximately the difference between the Hyades and the Pleiades.

In fact, the Coma Ber abundances are closer to those of Hyades stars which have a similar age, but a significantly higher metallicity (by $0.179 \pm 0.034 \mathrm{dex}$ ). The difference between the Coma Ber and Pleiades trends can only be explained by MS mixing, ruling out the standard model. The amount of mixing required is made uncertain by the errors in the cluster metallicities, but must give rise to around 0.3 dex of $\mathrm{Li}$ depletion at $6000 \mathrm{~K}$, rising to 0.8 1.2 dex at $5400 \mathrm{~K}$.

The discrepancy between Coma Ber and the Hyades, which was tentatively identified by J99 and which is now confirmed by our larger sample, seems far too small to be explained by standard PMS depletion. Any MS depletion of lithium should reduce the amount of $\mathrm{Li}$ in both clusters, without decreasing the abundance gap between them, unless initial rotation rates in Coma Ber were considerably larger than in the Hyades. The evidence shown in Fig. 2 does not support higher rotation rates in Coma Ber, although some convergence of rotation rates may have taken place over several hundred million years. If anything, we might expect MS mixing to amplify the difference between the cluster $\mathrm{Li}-T_{\text {eff }}$ trends, since the more metal-rich Hyades stars should destroy their Li more efficiently than those of Coma Ber. The Hyades is also 100-200 Myr older than Coma Ber allowing more MS mixing to take place. This suggests that Coma Ber and the Hyades started life on the ZAMS with Li abundances which were similar (at least for stars in the temperature range $5400<T_{\text {eff }}<6000 \mathrm{~K}$ ), and comparable with the level of Li seen in the Pleiades today. Again, this flatly contradicts the predictions of standard models but a lack of metallicity dependence for PMS Li depletion is also supported by observations of the young metal-rich cluster Blanco 1 (Jeffries \& James 1999) and the young metalpoor cluster NGC 2516 (Jeffries et al. 1998). Both of these clusters show ZAMS Li depletion patterns and rotation rate distributions similar to that of the Pleiades.

There are two possibilities remaining that could rescue the standard model of PMS Li depletion. The first is that the metallicities of Coma Ber, the Hyades and the Pleiades are incorrect. Whilst we believe the absolute $[\mathrm{Fe} / \mathrm{H}]$ values we have used probably have errors larger than those stated, the quoted errors probably do reflect the uncertainties in the relative abundances of the three clusters, because they were analysed using similar spectra, atmospheric models and temperature scales. The second possibility is that these clusters have non-solar abundance ratios. The metallicities we have used refer only to the iron abundance, whereas CZ structure, and hence Li depletion, is also significantly affected by the abundances of silicon, neon, magnesium and oxygen (Swenson et al. 1994). A detailed and consistent abundance analysis for all these elements in the relevant clusters would be invaluable. However, we consider this unlikely to be a saviour for the standard model simply because of the growing weight of evidence that cluster Li depletion patterns among $\mathrm{G}$ and $\mathrm{K}$ stars are ordered according to their age rather than their metallicity (Jeffries 2000).

\subsection{The cluster luminosity function and mass segregation}

In Sect. 5 of their paper, O98 examine the spatial and kinematic distributions of proper-motion selected Coma Ber candidates. They concluded that the cluster core is surrounded by a moving group of extra-tidal stars which share similar space motions but which are not gravitationally bound to the system as a whole. By separating the cluster candidates into two subsets (those objects within 5 degrees of the cluster centre, and those more than 5 degrees from it), it was shown that whilst the luminosity function falls sharply at $V=9$ in the centre, the moving group still appears to have a rising luminosity function at $V=10$ (see Fig. 4). The inferences from this are that the drop in the cluster-core luminosity function was real and not caused by incompleteness, and that the origin of the moving group is low-mass stars which have suffered dynamical segregation and evaporation from the cluster core. We could also surmise that even more low-mass members might be found among the moving group at magnitudes below the current limits of proper-motion surveys. Plots of both luminosity functions are given in Fig. 4a.

These conclusions hinge critically on the number of faint moving-group proper-motion candidates which are bona fide cluster members. There are only seven faint $(V>9.5)$ moving-group objects, (those which are more than 5 degrees from the cluster centre), and they have all had their membership credentials tested by radial velocity and $\mathrm{Li}$ abundance measurements in this paper. Four were found to be non-members (BD+16 2505, BD+25 2631, BD+36 2312 and TYC 25341715 1), while the three others (BD+21 2514, HD 114400 and HIP 61205) were confirmed as cluster members. There are also three faint cluster-centre objects (BD+28 2119, Tr 12 and $\operatorname{Tr} 141)$ which we have confirmed as members, J99 identified a further three stars with $9.5<V<10.5$ which also appear to be cluster members, yet which are not listed as such by O98: $\operatorname{Tr} 120$, Tr 132 and Tr 150. All of these would be classified as central-cluster members. This confirms that there is some incompleteness in the O98 sample and weakens the evidence for a sharp drop in the luminosity function at $V=9$.

We have performed a formal Kolmogorov-Smirnov double-sided test on the cumulative luminosity functions 

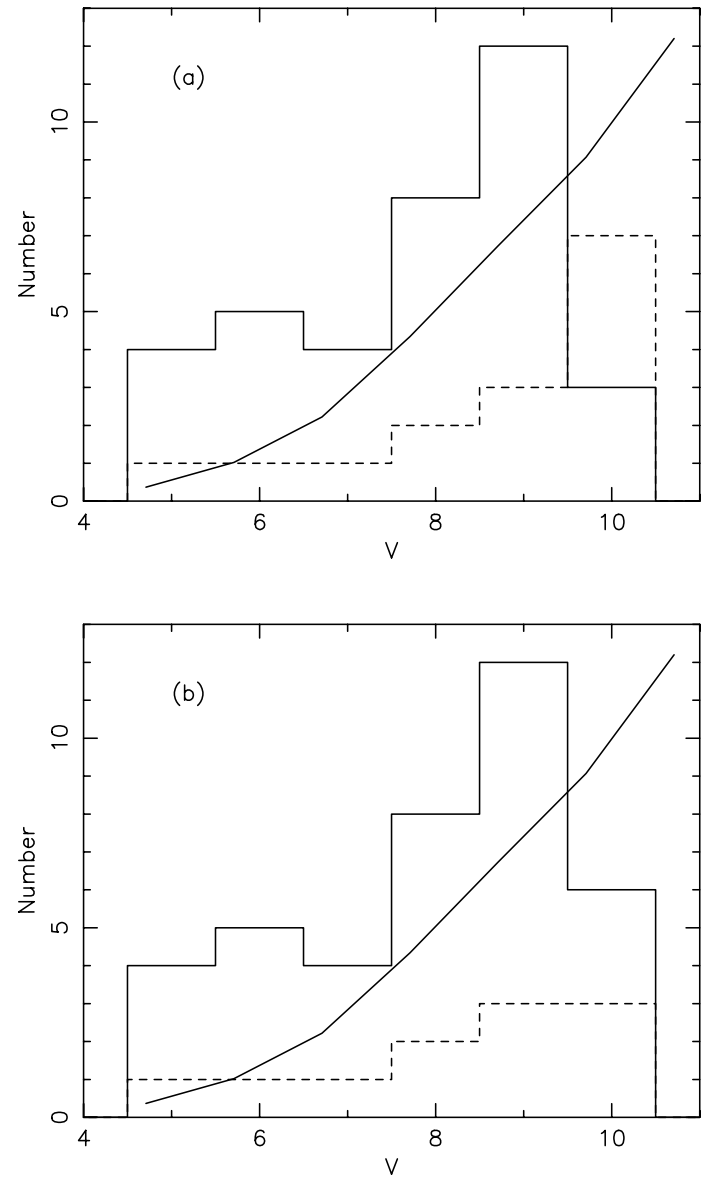

Fig. 4. Luminosity functions for the central parts of the cluster (solid histogram), the moving group (dashed histogram) and a normalised canonical field luminosity function from Miller \& Scalo (1979 - solid line). a) Comparison based on the original sample and classification presented by O98. b) Comparison after the samples have been modified to include new members and exclude new non-members found in this paper

of the central cluster and moving group from O98, using their membership classifications. We find that the distributions are different at the 92 per cent confidence level in agreement with their conclusion. However, if we remove the 4 stars which we classified as non-members from the faint moving-group sample, this confidence level reduces to 45 per cent. Inserting the three "new" members of the central cluster reduces this confidence level further, to only 11 per cent, i.e. we can conclude that the two luminosity functions are indistinguishable. One-sided Kolmogorov-Smirnov tests of these modified cumulative luminosity functions against the canonical field luminosity function given in Table 1 of Miller \& Scalo (1979, assuming a cluster distance modulus of 4.7 ), yield probabilities of 0.1 and 80 per cent respectively that the central-cluster and moving-group luminosity functions could be similar to those of field stars. Of course, these probabilities depend critically on whether the sample is more incomplete at fainter magnitudes. It would require adding a further 5-6 faint stars to the central-cluster sample to make its luminosity function indistinguishable from that of the field.
The implications of this are, depending on the level of incompleteness, that the luminosity function probably flattens, or even drops, for $V>9$ in the central part of the cluster. This is presumably as a result of dynamical evolution. However, there is no clear evidence that the evaporated or ejected stars form the moving group around the cluster, or that the luminosity function continues to rise among the faint moving-group members: its luminosity function could be consistent with either the field or the central part of the cluster. Therefore, it remains highly uncertain whether a significant number of moving-group members could be found at fainter magnitudes and lower masses.

\section{Conclusions}

In this paper we have obtained radial velocities, projected equatorial velocities and lithium abundances for a sample of Coma Berenices open-cluster candidates, which were selected on the basis of their proper motions. The radial velocities were used to refine the candidate list further. Of the 20 objects observed, 6 were found to be non-members, 1 is a spectroscopic binary, 1 is a probable spectroscopic binary, while the remaining 12 have radial velocities consistent with cluster membership.

By combining our data with the work of O98, Boesgaard (1987) and Kraft (1965), we have obtained a near-complete ( $>60$ per cent) membership census of F, G and early-K stars in the Coma Ber cluster. We have examined Li depletion in these stars, compared them with similar objects from the Hyades and Pleiades clusters and arrived at the following conclusions:

- Li depletion has occurred in the F stars of Coma Ber. The Li levels in these stars are close to, or slightly lower than, those of comparable stars in the Hyades, a cluster with a similar age but higher metallicity. Several lateF stars in Coma Ber have suffered 0.2-0.5 dex more depletion than similar stars in the Hyades. It is unlikely that these stars are non-members and we have no convincing explanation for this observation at present. We speculate that some Coma Ber F stars had a significantly different rotational history to those in the Hyades, although the rotation data in this paper and the literature do not show any peculiarities to support this;

- We have confirmed the tentative results presented by Jeffries (1999) with our larger sample of G and K objects in the Coma Ber cluster. We find that these stars have undergone less $\mathrm{Li}$ depletion than their counterparts in the more metal-rich Hyades, but significantly more depletion than younger Pleiades stars of similar metallicity. These observations contradict the standard Li-depletion model, consisting mainly of PMS convective mixing, since this would predict a strong metallicity dependence in Li-depletion patterns which we do not see; 
- The difference between the Pleiades and Coma Ber Li depletion at a given temperature could be attributed to non-standard mixing mechanisms, such as rotationinduced turbulence. If this is the case, such mixing must be responsible for $\mathrm{Li}$ depletion of around $0.3 \mathrm{dex}$ (at $6000 \mathrm{~K})$, rising to $0.8-1.2 \mathrm{dex}$ in cooler objects $(5400 \mathrm{~K})$;

- The similarity between the Hyades and Coma Ber Lidepletion patterns among $\mathrm{G}$ stars is difficult to explain with non-standard mixing, unless both clusters joined the ZAMS with Li depletion patterns similar to that of the Pleiades. Again, this contradicts our notions of how Li depletion and mixing work during PMS evolution.

Finally, we have used our data to re-evaluate some of the conclusions on the spatial distribution and luminosity function of proper-motion cluster candidates which were reached in earlier work. In particular we have found that, among the fainter stars, earlier membership samples were not complete and included a significant number of nonmembers. Taking this into account we find that the luminosity functions of stars in both the central cluster and in the proposed extra-tidal moving group of star are indistinguishable for $0<M_{v}<6$. This questions whether the moving-group is preferentially populated by ejected or evaporated low-mass stars from the central cluster. However, there is still evidence from the central-cluster luminosity function that preferential evaporation of lower mass stars has indeed occurred.

Acknowledgements. The William Herschel and Isaac Newton Telescopes are operated on the island of La Palma by the Isaac Newton Group in the Spanish Observatorio del Roque de los Muchachos of the Instituto de Astrofisica de Canarias. The authors acknowledge the travel and subsistence support of the UK Particle Physics and Astronomy Research Council (PPARC). AF and JRB were funded by PPARC postgraduate studentships. Computational work was performed on the Keele and St. Andrews nodes of the PPARC funded Starlink network. This research has made use of NASA's Astrophysics Data System Abstract Service and CDS' SIMBAD Astronomical Database.

\section{References}

Argue, A. N., \& Kenworthy, C. M. 1969, MNRAS, 146, 479 Balachandran, S. 1995, ApJ, 446, 203

Balachandran, S., Lambert, D. L., \& Stauffer, J. R. 1996, ApJ, 470, 1243

Boesgaard, A. M. 1987, ApJ, 321, 967

Boesgaard, A. M., \& Budge, K. G. 1988, ApJ, 332, 410

Boesgaard, A. M. 1991, ApJ, 370, L95

Boesgaard, A. M., \& Friel, E. D. 1990, ApJ, 351, 467

Böhm-Vitense, E. 1981, ARA\&A, 23, 19, 295

Bonifacio, P., \& Molaro, P. 1997, ApJ, MNRAS, 285, 847

Bounatiro, L. 1993, A\&AS, 100, 531

Carlsson, M., Rutten, R., Bruls, H. J. M. J., \& Shchukina, N. G. 1994, A\&A, 288, 860

Charbonneau, P., \& Michaud, G. 1988, ApJ, 332, 964

Deliyannis, C. P., \& Ryan, S. G. 1997, ApJ, 480, 43
Deliyannis, C. P., Boesgaard, A. M., Stephens, A., et al. 1998, ApJ, 498, L147

Duflot, M., Figon, P., \& Meyssonnier, N. 1995, A\&AS, 114, 269

Friel, E. D., \& Boesgaard, A. M. 1992, ApJ, 387, 170

Gray, D. F. 1992, The Observation and Analysis of Stellar Photospheres, 2nd edition (Cambridge University Press)

Haagkvist, L., \& Oja, T. 1973, A\&AS, 12, 381

Jeffries, R. D. 2000, in Stellar clusters and associations: Rotation, activity and dynamos, ed. R. Pallavicini, G. Micela, \& S. Sciortino (Astronomical Society of the Pacific Conf. Ser., San Francisco), 198, 245

Jeffries, R. D. 1999, MNRAS, 304, 821

Jeffries, R. D., \& James, D. J. 1999, ApJ, 511, 218

Jeffries, R. D., James, D. J., \& Thurston, M. R. 1998, MNRAS, 300,550

Johnson, H. L., \& Knuckles, C. F. 1955, ApJ, 122, 209

Jones, B. F., Fischer, D., \& Soderblom, D. R. 1999, AJ, 117, 330

Kraft, R. P. 1965, ApJ, 142, 681

Knude, J. 1989, A\&AS, 81, 215

Kurucz, R. L. 1993, Kurucz CD-Rom 13, Atlas 9, SAO, Cambridge

Lyngå, G. 1987, Catalogue of Open Cluster Data, 5th ed., Lund Observatory

Marilli, E., Catalano, S., \& Frasca, A. 1997, Mem. Soc. Astr. It., 68,895

Mermilliod, J.-C. 1976, A\&AS, 26, 419

Mermilliod, J.-C. 1981, A\&A, 97, 235

Michaud, G. 1986, ApJ, 302, 650

Miller, G. E., \& Scalo, J. M. 1979, ApJS, 41, 513

Odenkirchen, M., Soubiran, C., \& Colin, J. 1999, NewA, 4, 133

Oja, T. 1985, A\&AS, 61, 331

Perryman, M. A. C., Lindegren, L., Kovalevsky, J., et al. 1997, A\&A, 323, L49

Philip, A. G. D., Demarque, P., Sweigart, A. V., \& Ciardullo, R. B. 1977, PASP, 89, 554

Pinsonneault, M. H. 1997, ARA\&A, 35, 557

Pinsonneault, M. H., Walker, T. P., Steigman, G., \& Narayanan, V. K. 1999, ApJ, 527, 180

Radick, R. R., Thompson, D. T., Lockwood, G. W., Duncan, D. K., \& Baggett, W. E. 1987, ApJ, 321, 459

Rosvick, J. M., Mermilliod, J. C., \& Mayor, M. 1992, A\&A, 255,130

Ryan, S. G., Norris, J. E., \& Beers, T. C. 1999, ApJ, 523, 654

Saxner, M., \& Hammarbäck, G. 1985, A\&A, 151, 372

Soderblom, D. R., Oey, M. S., Johnson, D. R. H., \& Stone, R. P. S. 1990, AJ, 99, 595

Soderblom, D. R., Jones, B. F., Balachandran, S., et al. 1993a, AJ, 106, 1059

Soderblom, D. R., Stauffer, J. R., MacGregor, K. B., \& Jones, B. F. 1993b, ApJ, 409, 624

Soderblom, D. R., Jones, B. F., Stauffer, J. R., \& Chaboyer, B. 1995, AJ, 110,729

Swenson, F. J., Faulkner, J., Rogers, F. J., \& Iglesias, C. A. 1994, ApJ, 425, 286

Thorburn, J. A., Hobbs, L. M., Deliyannis, C. P., \& Pinsonneault, M. H. 1993, ApJ, 415, 150

Trumpler, R. J. 1938, Lick Obs. Bull., 494, 167

Urban, S. E., Corbin, T. E., \& Wycoff, G. L. 1998, AJ, 115, 2161

Ventura, P., Zeppieri, A., Mazzitelli, I., \& D'Antona, F. 1998, A\&A, 331, 1011

Wielen, R. 1977, A\&A, 60, 263

Yang, J., Turner, M. S., Schramm, D. N., Steigman, G., \& Olive, K. A. 1984, ApJ, 281, 493 